\title{
LIVED EXPERIENCES OF PNEUMONIA VICTIMS IN DAVAO CITY: A PHENOMENOLOGICAL STUDY
}

\author{
Stephanie Porras \\ Juvyneth Follante \\ Stephen Aseo \\ Ellamarch Lapayag \\ Angel Dominguiano \\ Jhon Escanda \\ Mark Dacuycuy \\ Kathleen Bandigan \\ Chantelle Del Rosario \\ Marites Dela Torre
}

March 2019

Correspondence:

\author{
Stephen Aseo \\ Team Leader \\ University of Immaculate Conception \\ Annex Campus \\ Bonifacio St., Davao City 8000 \\ Davao del Sur, Philippines \\ (082) 2271573 \\ (082) 2273794
}




\begin{abstract}
An access to primary care offered by the government has been the major concern of this study, which are deprived with complicated perceptions specifically in rural areas. The study engaged a phenomenological approach that characterized human experiences, and mainly to observe the behavioral response of people involved in this research. Also, it uses a purposive sampling technique that involves handpicking participants based on pre-selected criteria. The results gained specified themes through the use of thematic analysis. Based on the identified results, there were four themes introduced in the study that encompasses the problem; and the four themes that were mentioned were Financial Barriers, Structural Barriers, Cognitive Barriers, and Psychological Barriers. Each theme has sub-themes that mimic the problem of the community to the medical society within its access to health services. With regards to this result, the researchers came up with certifying alternatives that would develop the governments' capabilities in giving quality to health services. It was determined that people should be able to comprehend the barriers hindering them to access the health services. Thus, they should commit themselves on appealing an effective communication to the government. The lingering biases and prejudices of medical experts to the remote communities should be dissipated instantly to avoid future complications. In this similar way, the two should submit to an organized and to an effective stabilized relationship for a better response.
\end{abstract}

Keywords: Primary care, Pneumonia, Phenomenological approach, health services 


\section{INTRODUCTION}

\section{BACKGROUND OF THE STUDY}

According to Wafa Aftab et al. (2018), the central solution for curing children with pneumonia and diarrhea are seeking proper medication the moment the child is observed to be experiencing the symptoms of the illness stated above. Effective and proper medication prevented the majority of deaths due to pneumonia and diarrhea. However, it is observed globally that effective interventions for most cases are critically low. Facilities needed for treatment within high mortality areas is not adequately accessible, particularly within the critical first twenty-four (24) hours after the decease started. Unfortunately, many low and lower-middle income countries are still having problems exercising community-based health worker services for these childhood illnesses. Due to the inaccessibility to healthcare, many pneumonia victims are dying particularly those living in remote areas. To contextualize the statements above, the foremost element in eliminating any illness is to make sure those healthcares are accessible to anyone wherever they are in the globe.

There were 2951 children with WHO-defined clinical Pneumonia, of whom 1074 were severe or very severely ill, 278 were radiographic, and 219 were hypoxemic. While most children with Pneumonia were from urban barangays there was a disproportionately higher distribution of severe/very severe Pneumonia in rural barangays, and the most severe hypoxemic children were concentrated in the northern barangays most distant from the regional Hospital. According to USA Today Travel Tips, hospitals in rural areas of the Philippines typically offer far more limited treatment than in Manila and other cities. Healthcare providers and health educators are concentrated in urban areas like Metro Manila. Thus there is a death of healthcare of manpower in the Raleigh area (the manila times 2018) when it comes to healthcare in the Philippines availability and accessibility vary depending on where you are. Healthcare in the Philippines can get a bit hard in more rural 
areas. The hospital facilities in rural areas are usually basic, and not as well equipped as those in the urban areas. But rest assured that healthcare providers in many of the hospitals in the rural areas are trained to handle emergency cases. Effective on October 29, 2017, members of the Philippine Health Insurance Corporation (PhilHealth) will no longer contend with the minimum length-of-stay for community-acquired pneumonia in accredited facilities to avail themselves of their social health insurance benefits. The officials thought of some people faking their illnesses to stay longer in the hospitals. PhilHealth however required papers from the patients to prove their illnesses. Under all the case rates provider payment mechanism, PhilHealth pays 15,000 pesos for moderate risk pneumonia, and 32,000 pesos for high-risk pneumonia (PhilHealth, 2017).

As discussed by Silva and Johnson (2009), the most common thing that developing countries share is that they are struggling in improving their health care delivery system. In addition to that, they also do not have enough resources to act. Without having sufficient financial and human resources, a local government must weight which initiatives would benefit more the people in the community and implement it first. Often, social development initiatives, including health care, are de-prioritized. For many developing countries, the availability of the facilities in a clinic or hospital decides where a patient must go and seek for medication. Once the patient already decided, the facility's location and the factors it takes to reach it (i.e., travel time, travel cost) influence the choice of facilities available to the patient. The availability of the facilities the patient can use becomes fewer as it goes to more rural areas. Due to these barriers, some patients postpone going to the hospital until such time when their illness needed more medical attention.

Community-based healthcare interventions are done to provide immediate treatment to patients who are suffering from an illness. By performing the needed mitigations for an illness, the possibility of it progressing to severe levels are prevented, reduce household costs of seeking treatment, and fill the gap that may exist due to an inadequacy of health facilities, particularly in remote areas. Community-based interventions are highly recommended and are now 
widespread, which aims to increasing access to the areas that health systems are weak and underfunded. That justifies that inaccessibility to healthcare has a big impact on the patients surviving rate particularly in remote areas. Receiving proper medication and access to healthcare is first in treating illnesses (Matuvo, Nanyiti, and Rutebemberwa, 2014).

Primary health care considered a stepping stone toward the elimination of Pneumonia, but it does not guarantee a hundred percent that it will be diminished as soon as possible. The authorities are doing everything they can to put an end to this illness, but up until now, the government is still having their lapses towards this problem. And various studies have proven those lapses, for instance, Wafa Aftab et al. (2018), and Silva and Johnson (2009). In this research, the researcher wanted to evaluate if pneumonia victims in rural areas received or undertaken any primary care from the government. This response would lead to a good cause and would benefit the participants and researchers in this qualitative study

It was difficult for people who lived in far-flung areas to have health care assistance given by the government. The people living there have experienced the struggles of getting medicines prescribed by physicians about their health problems. On this circumstance, the researchers guaranteed an exceptional study that would compromise the needs of the community, especially with this epidemic. The researchers have indeed picked the title to stimulate the best course action for this cause. With this purpose in mind, the researchers prepared questions that would help them to collect reliable and authentic information from the participants. The researchers do believe that this problem needs an immediate response. And of course, this would benefit the two parties. Otherwise, both would suffer a long time regression towards one unaltered decision.

\section{PURPOSE OF THE STUDY}

The sole objective of this phenomenological study was for the researchers to undergo research if they have access to primary health care given by the government for Pneumonia victims. While the research was going on, there were 
specific objectives that cover under the research. The researchers would like to determine if the participants received or undertaken any health assistance from the government before, and if not what are the barriers that inhibit the best course action for Pneumonia victims. Also, the researchers should be able to describe on how they manage to cope with Pneumonia. Moreover, the researchers wanted to reach the focal conclusion for a better response and specific needs of the said pneumonia victims.

\section{RESEARCH QUESTIONS}

The researchers would like to discuss the lived experiences of Pneumonia victims in rural areas in Davao City in accessing the primary health care given by the government. Also, for them to complete this research and derive new knowledge from the data gathered, the researchers seek to answer the following question:

1. What are the lived experiences of the Pneumonia Victims?

\section{THEORETICAL LENS}

This phenomenological study was anchored in several theories such as The Health Belief Model, The Transtheoretical Model, and Social Cognitive Theory for the researchers to investigate the problem of Primary Health Care of Pneumonia in rural areas.

The Health Belief Model (HBM) was developed by social scientists at the U.S. Public Health Service to understand the negligence of a person to adopt the strategies for preventing diseases or screening test for early detection of diseases. The HBM proposes that the belief of a person about a personal threat of an illness or disease along with the person's judgment on the efficiency of the suggested health behavior or an action that will predict the person to adopt the behavior. The Health Belief Model justifies that no matter what the government does, it still depends on the person if he/she avails the programs that the government has prepared for them. To contextualize this statement, the researchers can say that if 
a certain community has access to the primary care given by the government but does not pay much more attention to it, it is more likely for them to endure more of the illness. (LaMorte, 2018).

The Transtheoretical Model (TTM) also called as the stages of Change Model (Prochaska and DeClemente, 1970) The Transtheoretical Model (TTM) centers around the basic leadership of an individual. The model works on the suspicion that individuals do not change practices rapidly and conclusively. TTM is not a hypothesis yet a model; distinctive conduct speculations and builds can connect to different phases of the model where the individual think it might be the best change in their conduct. It does not require time to what extent an individual can stay in his behavioural conduct. These procedures result in methodologies that assistants the individuals make and look after their changes in conduct. The Transtheoretical Model gives recommended systems or general well-being mediations to address individuals at their basic leadership process. The model accepted that people make intelligible and legitimate plans in their basic leadership process when this is not in every case genuine (LaMorte, 2018).

As cited by Glantz et al. (2002), The Social Cognitive Theory is said to be pertinent to health communication. This theory illustrates how people develop and conserve certain behavioral patterns, while also providing the basis for intervention strategies (Bandura, 1997). This theory justifies that there are factors that can greatly affect ones' behavior. In a community that has a lot of Pneumonia victims and the majority of the residents in that community do not consider the value preventive measures. There is a big possibility that other residents will be influenced by that kind of reasoning, and because of that, they would be considering a delay in seeking proper medication.

\section{REVIEW OF RELATED LITERATURE}

\section{PRIMARY CARE}

In the United Kingdom, a review of access to healthcare found that the people living in rural areas, the elderly and those socioeconomically disadvantaged 
groups have lesser access to health care. The possible explanations for this may include geographical isolation with one out of older people in rural areas that are living more than 4 kilometers away from their primary healthcare provider, poor transport availability, one out of three pensioner households does not have any access to a car, and the association of deprivation. In the triple jeopardy of age, both of rural and deprivation causes the increased morbidity, but it decreased the access; an example of a well-known 'inverse care law' it states there that healthcare provision is inversely to need. Access is a complex concept leading to several previous studies using service utilization as a proxy endpoint. This is conceptually different from access because an individual could have good access but may never need to use a service.

As elaborated by the British Medical Journal Open (2015), in the United Kingdom, they have an aging population of 65-year-olds set that increases by 9 million over the next 35 years just like most of the high-income countries. An aging number also presents lots of challenges, such as the increasing number of people with chronic health problems and the inescapable impact on healthcare resources. Kirchoff et al. (2014), stated that the magnified demand for medical services expected to result from the Affordable Care Act (ACA) may be felt unequivocally in rural areas. While study have found that the standard of care delivered to rural patients is nearly as good or highly accessible in the urban areas, the belief persists that finest medical aid is just accessible in big cities. However, another investigation appearing in the National Rural Health Association's Journal of Rural health finds few significant variations between the rural and urban medical aid physician on key measures of expertness as well as their attitudes about participation in quality care improvement. The study did notice variations in the likelihood of the physicians' who was impaired or incompetent, in their confidence evaluating new data and in many aspects of their interactions with the patients.

Campbell (2014), states that he found out those rural and urban physicians were more alike that they were different in line with professional beliefs and behaviors. Despite the results and other evidences that they have gathered, 
perceptive view still occurs that rural primary care was not consistently active like the urban cities. They need to learn more about factors contributing to the misconception and role that may play in the continuing shortage of rural doctors in the United States. Moreover, Campbell et al. (2014), noted that, whereas smaller rural communities might have disadvantages in terms of fewer training choices, rural primary-care physicians are considerably more likely to participate in activities like quality improvement in their practices and hospitals. The fact that rural physicians are more likely to have personal as well as skilled relationships with their patients may give them a better sense of environmental and lifestyle factors that affect patients' health might offer them a challenges when adept responsibilities contradiction with patient expectations and perceptions.

On the other hand, there were no important variations between rural and concrete physicians' attitudes concerning participation in quality improvement activities and also the importance of open communication with patience, including reporting any errors in their care. Rural physicians were more likely to participate in error-reduction initiatives, in reviews of other physicians' records, and to feel ready to contribute to quality improvement efforts. Additionally, they were more likely to agree that physicians ought to discuss the cost of care with their patients and to report having supplementary health care or uninsurable patients to their patient panels throughout the preceding year. Anne Kirchoff (2014), discussed that rural doctors were serious in contributing high-quality care and they were committed in supporting safety-net patients. In addition to that response, the Affordable Care Act should be able to help more rural primary care providers receive payments for the care they were giving without charge. Although both urban and rural physicians united on the importance of reporting colleagues who were incompetent or in a way impaired, rural physicians were considerably more like to point really knowing about such individuals. Similar percentages of both rural and urban doctors felt ready to accommodate impaired or incompetent colleagues. Rural physicians were more likely to report having consummated patient requests for brand-name medicine once less expensive generics were accessible a 
common measure of wasteful medical practice and were less likely to feel ready to gauge new medical information.

Previous study states that there are a lot of diseases which leads a person to its final breath and that includes pneumonia. Pneumonia is one of the diseases leading cause of death in infants and young children in developing countries, including Philippines. Nonetheless, there are only limited data at the community level which makes them to hold a household level interview survey in Biliran Island, Philippines, where the caregivers were interviewed with the use of a semistructured questionnaire to check if the children had symptoms suggesting pneumonia-like episodes from June 2011 to May 2012. One out of ten children less than the age of 5 years old develops pneumonia- like episodes annually in Biliran Island, Philippines. Some risk factors, including the age of a child, the gestational age at birth, history of asthma, socio economic status (SES) and even the travel time to a health care facility are notably related with the occurrence of pneumonia-like episodes, but the factor between pneumonia development and risk factors such as history of asthma remains evasive. Better access to the health care facilities helps to improve a health care-seeking behavior and they would also help to aid early and to teach effective management to the patients with pneumonia (Kosai et al, 2015).

\section{PNEUMONIA}

Pneumonia is a life threatened disease and it is also one of the most dangerous global health problems in children, especially in those countries who is still developing. According to the annual reports from its Department of Health $(\mathrm{DOH})$ pneumonia is ranked as the number one leading cause of death in children those ages from 1 to 4 . Furthermore, the Integrated Management of Childhood IIIness (IMCl) which is developed by the World Organization Health and the United Nations Children's Fund, nurture a better and effective patient management in order to lessen the rate and cases of disease in a population which is caused by some common childhood diseases, including pneumonia. The Millennium Development Goal 4 (MDG 4) of the United Nations objective is to lessen the cases 
of diseases especially the pneumonia by two-thirds by 2015 from 1990 level. Nevertheless, many of the developing countries are not on track to accomplish The Millennium Development Goal 4 (MDG 4). There are many factors that hindering the achievement of MDG 4. Including pneumonia, it remains to cause 1.4 million deaths of children annually under the age of 5 , accounting for $18 \%$ of universal mortality in this age group globally. Around 110 per 1000 person per year is the estimated incidence rate of pneumonia in children whose age is less than 5 years old in the Western Pacific Region.

In relation to the study, previous results found out that there is a close interrelation between childhood pneumonia and risk factors such as age, sex, parents' educational level, parental smoking, number of siblings, type of cooking fuel, type of toilet facility even the socio economic status (SES). Remarkably, the above-mentioned studies were conducted in the hospital settings. When children develop pneumonia, caregivers may not always take them to the hospitals, especially in the countries with limited resources, due to some numerous reasons, for instance, financial problems, lack of knowledge, or even poor accessibility to healthcare facilities. Data on the health care-seeking behavior of pneumonia, specifically at the community level is needed, in order to understand the real situation of pediatric pneumonia, but the said data are still limited. A qualitative data was collected from 63 older and younger mothers and it revealed that most of them recognized pneumonia and was able to describe mild and severe signs and symptoms to explain incidence of pneumonia. Respiratory illness was attributed to humoral imbalance, supernatural causes and "negligent" mothers. The role of the family was important in decision-making. Rural mothers were relieved and satisfied to be able to have a quick access to low-cost medicines from Bangladesh Rural Advancement Committee (BRAC) health volunteers, who is clearly responsible for health care practices. An In-depth interviews and focused group discussion was conducted with 23 health volunteers and after the said activity it showed that all 22 health volunteers were able to correctly identify breathing rates and their association with pneumonia. 
As cited by the Bangladesh Rural Advancement Committee (BRAC), they have been working in the field of socio-economic development and the BRAC launched an intervention program to decrease the number of ARI-related deaths in rural Bangladesh. The Acute Respiratory Infections program works through a system of community-based education, detection and control programs. Through educating the people around the community about pneumonia it will surely help them recognize mild and severe signs and symptoms of pneumonia. Educating them now know on how to identify the illness, we can now proceed to the second step which is detection. Given that they are knowledgeable about the illness already it is now easy for them to conclude that a person suffer from pneumonia. Lastly are control programs, through this people in the community especially the caregivers will surely know how to act in the patients' current situation. The study indicated that mothers were aware of Acute Respiratory Infection and familiar with its signs and symptoms. Mothers in that community care for their ill child at home or seek for proper medical care depending on the interpretation of illness. Health volunteers were also knowledgeable about Acute Respiratory Infections. Despite limitations in supervision and technical support, the program is firmly bonded with the community with volunteers positively educating rural women's health care practices. Through these results you can see that well educated community members and volunteers can greatly affect the morbidity and mortality within the community (Rashid et al, 2001).

The Acute respiratory infections (ARI) are considered to be a major cause of mortality and morbidity among young children in developing countries. Every year an estimated 2.2 million children die from infections of respiratory tract, principally pneumonia, worldwide. ARI, particularly acute lower respiratory infections (ALRI), is accountable for nearly a third of all deaths of children under five years of age in many countries. Insufficient and delaying medical care results to a large number of children dying each day from ARI in Bangladesh. For cases of severe ALRI, such as pneumonia, fast and adequate medical care is essential for survival. Research findings exhibited that educating communities and increasing awareness of ARI helps a lot of people specially those who are 
experiencing or to those to have love ones suffering from that illness. An effective control program involves three main parts: early detection and case management, vaccinations, and health education. Effective case management depends on how you disseminate information to the community members, which encourages people to use proper health care facilities and seek medical attention as soon as possible to avoid serious complications. Cultural understanding and practice of the community needs to be carefully contemplated when developing precautionary measures and program for improving the knowledge and resilience of the community from the illness. A lot of families in the rural community have small knowledge about the said illness also on how to manage such life threatening situations, and many families in the rural community prefer traditional methods of treatment than seeking proper medical care.

Pneumonia is a disease where the lungs are infected due to bacterial, viral, or fungal infection. The lungs become inflamed, and the tiny air sacs, or alveoli, inside the lungs fill up with fluid. Pneumonia usually occur on a healthy person or to those in young but also it is very dangerous to old adults, infants, and those who have other diseases and impaired immune systems. If a person has a cold or flu then develops a high fever, chills, and cough with sputum he or she suffers from a lung infection disease called Pneumonia. It is a not a single disease and a very serious disease in lungs with the lots of possible causes. It tends to more serious for infants and young children, older adults, people who have other chronic health problems, and people who have weak immune systems as a result of diseases and other factors. Understanding the cause of pneumonia is very important because pneumonia treatment depends on its cause. Most pneumonia is contagious. Both viral and bacterial pneumonia can spread to others through inhalation of airborne droplets from a sneeze or cough. But while you can become infected with fungal pneumonia from the environment, it doesn't spread from person to person. There are several symptoms of pneumonia.

The first symptoms of pneumonia usually resemble those of a cold or flu. The person then develops a high fever, chills, and cough with sputum Crosta 
(2017). If a person has a fast heartbeat, diarrhea, sweating, headache, cough, abnormal breathing and many more he or she may have pneumonia. Those common symptoms of pneumonia depend on other underlying conditions and the types of it. Pneumonia can affect the human body by making the immune system weak and hard to filter germs out the air by breath. It is often spread via coughing, sneezing, touching, or even breathing, ad those who don't exhibit symptoms can also spread the illness. It is better to visit a doctor about the signs or symptoms that a person experienced. The doctor will provide a better meditation for the patient. A doctor will ask about the symptoms and medical history and will carry out a physical examination. Your doctor will also likely order a chest X-ray. Typically, pneumonia can be diagnosed with the physical exam and the chest $X$ ray. But depending on the severity of your symptoms and your risk of complications, your doctor may also order one or more of test.

The treatment will depend on the type of pneumonia a person have, how severe it is, and his general health. Pneumonia can be classified or characterized in different ways. Health care professionals often refer to pneumonia based upon the way that the infection is acquired, such as community-acquired pneumonia or hospital-acquired pneumonia. In the developing world today, many deaths from pneumonia are also preventable by immunization or access to simple, effective treatments. The treatment of Pneumonia depends on its type. Bacterial types of Pneumonia are treated by antibiotics. Viral types of Pneumonia are usually treated by plenty of fluids and the fungal types of pneumonia are usually treated by fungal infection. The doctors prescribe an OTC medication that helps to reduce fever, aches and pain, and suppress cough. Antibiotic, antiviral, and antifungal drugs are used to treat pneumonia, depending on the specific cause of the condition. Most cases of bacterial pneumonia can be treated at home with oral antibiotics, and most people respond to the antibiotics in one to three days.

To prevent pneumonia the patient may get pneumonia vaccine or flu vaccine. The pneumonia vaccine may reduce but doesn't eliminate the risk of pneumonia. It may have side effects that may affect the patient. Some people 
should not get the vaccine especially the children. But there are some ways to prevent pneumonia instead of getting the pneumonia vaccine. Healthy habits help to keep the immune system strong. Good hygiene and being hydrated is also important. Most people recover from pneumonia in one to three weeks. Resting and drinking a lot of fluids can help to recover quickly from pneumonia. The most common causes of Pneumonia are bacteria and viruses. They can cause the infection. There are also a few non-infectious types of pneumonia that are caused by inhaling or aspirating foreign matter or toxic substances into the lungs. It can be passed through by coughing or sneezing. Those most at risk of Pneumonia are aged under 5 years or over 65 years old, also those who recently recover from cold, who smoke tobacco and consume large amount of alcohol, who experience malnutrition and many more. Pneumonia is generally more serious when it affects older adults, infants and young children, those with chronic medical conditions, or those with weakened immune function. Bacterial, viral, aspiration, and fungal are the different types of pneumonia. Bacterial pneumonia can attack anyone, at any age. It can occur if he or she has a flu or cold. The viral pneumonia is caused by influenza virus may be severe but sometimes fatal. This pneumonia is most serious in people who have pre-existing heart or lung disease and the pregnant women. The most at risk in this type of pneumonia are the children where most viruses attack the upper respiratory tract.

The aspiration pneumonia is a type of pneumonia where it has a complication in pulmonary aspiration. Chest pain, shortness of breath and difficulty of swallowing are one of the symptoms of aspiration pneumonia. The last one is the fungal pneumonia is the complication in the lungs caused by fungi. To prevent those different types of pneumonia, there are two vaccines for pneumococcal disease, the most common bacterial cause of pneumonia. These two vaccines are pneumococcal conjugate vaccine and a pneumococcal polysaccharide vaccine that is recommendable for both young and adults depending on their health conditions Crosta, P. (2017, November 27). 


\section{METHODOLOGY}

\section{RESEARCH DESIGN}

This is a qualitative study that uses a phenomenological approach to collect important and beneficial data. Understanding and interpreting the meaning that the participants gave to their everyday lives is what this phenomenological approach concerns (De Vos, 1998). The qualities of human behavior are what focus on the qualitative approach (Ferreira, Moutun, Puth, Schurink \& Schurink, 1998). The goal of such method is not to generalize, instead to understand and interpret the meaning and intention that underlie everyday human actions (Bailey, 1987; Bogdan \& Taylor. 1975; De Vos, 1998; Ferreira et al., 1998).

Phenomenological research is a method with strong philosophical origins, which can sometimes be challenging for novice investigators. Phenomenology is one of the methods of qualitative research that aims to depict how human beings experience a certain phenomenon. A phenomenological study attempts to set aside prejudices and preconceived assumptions about human experiences, feelings, and responses to a particular situation.

\section{SAMPLING METHOD}

Purposeful sampling which was also known as a purposive and selective sampling is the most common sampling strategy. In this technique, the participants are picked or sought after, depends on pre-selected criteria based on the research question (NawalSalim AL Kathiri, 2010). Participants were picked particularly by the researchers to fulfill the needed information to complete the study.

\section{PARTICIPANTS}

When the researchers identified the prospective participants, they approached and asked them to take part in the study. The participants were provided with the information sheet and were further briefed about their right to refuse in participating 
in the interview, and that participation was voluntary. When they agreed to participate, the participants were also briefed about their right to withdraw from partaking in the interview. They were further informed that all the information they gave to the interview is treated with confidentiality; the participants were not required to disclose their identifying details. Finally, the consent forms were handed to the participants for their participation and for the audio tape recording for spontaneously giving their consent to take part in an In-Depth Interview (IDI).

The researchers chose three (3) purposively chosen participants in rural areas of Davao specifically in Calinan and Malagos using purposeful sampling, whose ages eighteen (18) and above. The participants were selected because the researchers saw that they have experiences and insights about accessing primary healthcare of pneumonia victims in rural areas. Therefore, it was expected that the participants provided a meaningful description and validated data based on their experiences and responses.

\section{RESEARCH INSTRUMENTS}

The researchers of this phenomenological study prepared research questions. Research questions were made by the core topic of this study. Questions undergone evaluation and validation process presented to the researcher's teacher, Mr. Josepth Dave M. Pregoner. Until the validation was done, questions will be thoroughly checked for testing. The questions were used throughout the whole duration of the interview, and the researchers recorded the information from the participants. The English language was used as the medium in the making of research questions. Also, the researchers and participants used vernacular language during the interview for them to communicate effectively.

\section{DATA COLLECTION METHOD}

Face-to-Face individual interview was being used to collect data. According to Ferneria et al. (1988), the most important instrument in collecting data is interviewing. The interview was semi-structured, wherein a lot of questions and problems that needs to be discussed were constructed before the interview. The 
semi-structured interview gave room for further discussion, clarification, and investigation for some important and essential issues that unexpectedly arose during the interview. The researchers were allowed to read the non-verbal communication and reaction in a face-to-face interview, which can help analyze the data. The interview was audiotaped and later transcribed.

In relation, the researcher observed proper decorum in terms of psychological involvement that could "affect the validity and the reliability of the data" (Ferreira, et at., 1988: 147). Moreover, Bogdan and Taylor (1975), added that the interviewers were the ones who were responsible for creating a welcoming atmosphere in which the participants would feel comfortable enough to share freely and openly. This was attained by allowing the participants to partake in choosing a fitting and facilitating venue for the interviews.

\section{DATA ANALYSIS METHOD}

The researchers used content analysis to analyze the data which was gathered from personal interviews. According to Moore \& McCabe (2005), this is the type of research whereby data gathered categorized in themes and sub-themes. The main advantage of content analysis is that it helps data collected being reduced and simplified. However, human error is highly involved in content analysis, since there is a risk for researchers to misinterpret the data gathered, thereby generating false and unreliable conclusions (Krippendorff \& Bock, 2018).

\section{ORGANISING DATA:}

The deciphered data was scrutinized for the researcher to be acquainted with the information.

\section{GENERATING CATEGORIES, THEMES, AND PATTERNS}

This was a phase that required creative and analytical reasoning. The researcher at that point recognized essential themes, repeating thoughts, and patterns of beliefs, which helped the combination of the outcomes. The process of category generation included noting patterns in the research participants. As classes of 
importance rose, the researchers sorted those that were inside steady yet unmistakable from one another. Examples, subjects, and classes were revealed.

\section{TESTING EMERGENT HYPOTHESIS:}

When the researchers noticed the patterns and categories between them in the data, these developing hypotheses were then assessed to measure its credibility then the researchers tested them upon the data. This involved the assessing of the data for their informal adequacy, credibility, accountability, and assurance.

\section{SEARCHING FOR ALTERNATIVE EXPLANATION:}

When patterns and categories emerged in the data, the researchers engaged in challenging the patterns that seemed apparent. Alternative explanations were contemplated thoroughly until the researchers reached the explanations that are most logical among all.

\section{REPORT THE RESULTS:}

By relating to the acquired results of the report, the researcher then gave configurations, illustration, and meaning to the accumulated raw data.

\section{TRUSTWORTHINESS OF THE STUDY}

The aim of the trustworthiness in a qualitative inquiry was to support the arguments and data gathered by the researchers. Trustworthiness was established as to how the researchers conducted the procedures that need to be completed. In any qualitative project, four issues of trustworthiness demand attention and these were the following: the Credibility, Transferability, Dependability, and Conformability.

To establish credibility, the results must be credible or accurate from the viewpoints of the participants in the research. The qualitative research should be described or understood thoroughly the phenomenal interest from the position of the participants. Guba and Lincon (1985), established that the only one who can legitimately judge the credibility of the results were the participants. To establish transferability, Lincon and, Guba (1985), assert that it was the researchers' task to 
provide sufficient contextual information and background on the conducted research. The one who was responsible for doing the generalizing was the transferability. Geertz (1973), suggests that research should not have only factual information, but also to give a clear and strong explanation. The researchers provided a clear and enriched detail, on account of field experiences. To establish dependability, Lincon and, Guba (1985), manifest that the researcher should employ inquiry audit. In this research, the researchers have an outsider researcher who was not part of the research team to examine and observe both the process and product of the research study. This outsider researcher will be stated thoroughly how the data was gathered, how the researchers managed the arising problems, and how researchers came up with their decision throughout the research inquiry. To establish conformability, the researchers were confident that the research was done objectively and will never be influenced by personal feelings in representing facts. According to Lincon and Guba (1985), conformability is defined as to the degree to which the results could be confirmed or validated by others. The researcher can record the procedures for checking and re-checking or doing proofreading throughout the study.

\section{ETHICAL CONSIDERATION}

After participants were identified, there were several concepts that must be implied when researching on every participant. Their right when exposing themselves were also valuable where they are most likely vulnerable to some dangers. Researchers made sure their personal information and interest will be monitored to safeguard entrusted information. The Panel on Research Ethics (2010) elaborate on the key concepts of building a strong foundation of trust between the researchers and participants. Privacy, wherein researchers guaranteed the individual's right to be free from intrusion and interference by others. Proponents in this research considered the utmost comfort of participants as they engaged in such an interview. The informed consent was distributed to the participants to see if they agreed to the terms and conditions of the interview. Confidentiality, the use of transcripts and recordings for the whole duration of the interview, as the required 
materials for data gathering, will be held confidential. Authorization was needed to avail such information from the participants. Security, the information that was collected needs to be protected from other threats such as anonymous bystander near the research area or the place of interview. Identifiable information, which can be considered a threat to the participants, and people, may be expected to identify an individual, alone or in combination with other available information. Through these key concepts, the participants may be able to accept the offer comfortably presented by the proponents of this research, and in respect to the ethical consideration that was portrayed.

\section{ROLE OF THE RESEARCHERS}

The researchers in this study have an important role that needs to be observed since they followed a research procedure to have a smooth and orderly process. Researchers made sure that their personal feelings and biases will be set aside throughout the study. Thus, researchers focused on gathering data and interviewed to obtain more authentic information. The researchers provided questions, certainly to gain an insight into the participants' stories and experiences. They recorded the results and will find the central subject of the study. In addition to that response, researchers cautiously read and re-read the records that they have acquired from the in-depth interview. Furthermore, they analyzed and coded the data thoroughly that was being collected.

\section{RESULTS AND DISCUSSION}

This chapter has been formulated to answer the research question keeping the literature review and research findings from the interviews in consideration.

Research Question 1: What are the lived experiences of Pneumonia victims?

The participant's extracts generated four themes and several sub-themes to intersperse the findings of the study. Table 1 highlights the themes and subthemes generated in the study. 
Table 1. Thematic analysis

\begin{tabular}{|l|l|}
\hline Themes & Sub-themes \\
\hline Financial barriers & Health insurance \\
\hline & Employment status \\
\hline & Affordability \\
\hline & Bribery \\
\hline Structural barriers & Competing priorities \\
\hline & Service availability \\
\hline & Accessibility \\
\hline & Waiting times \\
\hline Cognitive barriers & Adult vs. children \\
\hline & Healthcare professional attitude and mentality \\
\hline & Language and communication \\
\hline & Discrimination \\
\hline Psychological barriers & Health literacy \\
\hline & Mistrust \\
\hline & Hopelessness \\
\hline & Fear \\
\hline & Anxiety \\
\hline
\end{tabular}

\section{Financial barriers}

Multifactorial components of this barrier exist and are a major determinant for ability to access healthcare. Health insurance is a crucial barrier; without insurance, primary care cannot be accessed therefore, secondary care referrals cannot be made, and only emergency care can be sought.

"...because she doesn't pay the, insurance, she doesn't have any insurance, she doesn't have family doctor." 
Health insurance is not required to access private care; however, payment is necessary which is mostly unaffordable to families with pneumonia victims. Some of the participants state that they managed to overcome having no insurance by creating a relationship with a doctor or any other medical professional who agreed to consult with them regardless of insurance status. However, they accepted that this relationship did not guarantee any other healthcare access points indicative of having insurance, such as secondary care referrals or subsidized prescriptions.

"One healthcare provider sent me to the hospital to be checked in 3 times, but at the hospital, they say I don't have insurance so they cannot accept me."

Employment status was shown to play a large role as unemployment is widespread within the community and impact upon the ability to acquire health insurance. All participants indicated that they have had issues with the ability to pay for either services or medicine - regardless of ability to access a doctor.

"... and it's another aspect for these families, some of them they don't have a job, and they don't pay their medical insurance, so they can't have a medical doctor, and they can't afford to pay."

Affordability extends to investigations and type of service desired. Pneumonia medical care was commonly needed, but the services are not covered within health insurance policies and costs are too high for most. Bribery and corruption within the healthcare sector was described by all but one interviewee. This did not solely involve the financial implications of paying bribes, but also impacted the standard of treatment and attitude received from healthcare professionals. Participants acknowledged that for a community who already cannot afford healthcare, additional bribery payments add to their problems and emotional stress.

“...she knows that God says not to give bribes, but if you don't give bribes in the hospital, they will let your kids die ... I would have to sit in the hallway until I have paid them something, they wouldn't let me in until I have paid them something." 
With limited income, competing priorities of money controls whether participants access the necessary care. Both cohorts explain that prioritization extends to everyday life and community members prioritize commodities like food or heating, or choose to invest in their children - rather than look after their own health.

"For example, we had a project and for those who participated, like $80 \%$ from the, the parent's meeting, they received some money, and I asked one of these ladies, if she puts some money, just for herself to, in order to have a medical check-up and she said oh no, I just I bought food, for heating, for heat, yeah and I bought some clothes for my kids and I'll pray to god to help, to have mercy on me."

\section{Structural barriers}

A general response amongst participants was a belief that healthcare services were plentiful, but not all were accessible, despite health insurance status, as many are provided by the private sector. Nevertheless, those with insurance face fewer problems. However, some participants believe the healthcare services available in there are not substantial for the needs of the community.

"The system is, is still antiquated, and under developed for the needs that they have."

One participant discussed how quality care can be accessed if one has more financial resources. Although this was in contrast to most participants who believed that service availability within the rural area was substantial, but the services are of a poor quality. It was found, as described by the first participant, that when a serious health issue occurred, they tried or wished to access care in an alternative area as the perceived quality of services was higher, showing an inequality of access depending on the location of a person's inhabitancy.

"if you want something good, better than [named own area], you go to other cities like [named area] or [named area]."

Service availability is thought by the community to be influenced by the prevalent staff shortages and salary disputes within the healthcare sector across the country. 
"The doctors are, are not paid enough to do their job, their salary is quite low, low, that's why they are not interested in being more responsible and being more involved and doing their best."

Some participants also explain how a service may not be available in their area and the only option is to travel elsewhere. However, travelling to another city is often not feasible because of waiting lists and the costs involved. Within the community area itself, participants described the multiple options of transport and the ease of travelling to services. Consequently, participants believe that geographically, there is good accessibility of services within the city. However, accessibility of care as an adult versus a child was considered more difficult, as children up to the age of 18 are theoretically entitled fast healthcare attention.

Nevertheless, children's treatment or medicine plus services out-with health insurance still needs payment, causing similar accessibility problems experienced by adults. Furthermore, barriers of queues or waiting times were considered to affect adults and children equally. Within this community, if a participant is placed on a waiting list to see a specialist or receive treatment, this was perceived as a positive step in accessing care. However, queues within hospitals, especially for those solely eligible to seek emergency care, pose a hurdle.

"So, they gave me just one tab of medicine to calm down, after this I left, because I had to wait for hours and hours and hours there, it is very crowded in there."

\section{Cognitive barriers}

Fear of negative healthcare professional attitudes and mentality due to previous bad experiences was shown to cause avoidance of care or premature departure from hospitals without receiving adequate help. Interview accounts portray doctors and other medical professionals ignoring, shouting and speaking derogatorily towards patients.

"Many times, I went to emergency, and there were times I got mad and just leave the emergency because nobody pays attention to me."

These attitudes were seen to alternate depending on situational circumstances. As such, the attitude and judgement received from healthcare 
professionals showed to correspond to the vulnerability status of those in the community for example, being poor, uneducated or impoverished and thus transpired as vulnerable population discrimination.

"There is also issues in this part of town, where doctors treat this group of people differently, or they view them differently, because of poverty, because they have low education, because they have no money and that influences things as well."

Despite this, participants' account of mistreatment from health professionals was also uncovered, as shown by the first participant. Other accounts state that doctors believe to view themselves as superior and to not treat patients humanely.

“The people don't speak nice with us and that's a problem. Because when we have a health problem like pneumonia, the first thing we think is not to go to a doctor because we are frightened, because we are scared, we will not talk with them or something like that."

Language and communication barriers involved the use of medical jargon and language that the patient is unable to understand, negatively impacting the doctor-patient relationship and resulting in the consultation being misunderstood. Negative discrimination was shown to extend further than just socioeconomic factors. There is a large percentage of ethnical population within Davao City, and many participants described cultural discrimination based on the stigma attached to this ethnicity.

"...there is even more resistance ... there you know, racism in our culture in general, because we are poor, we are going to be err, more marginalized because of lack of education, more marginalized, and there is already a mentality of if you're the doctor then you are superior."

It is suggested by one participant that pneumonia victims can be treated better if they have money, linking to healthcare professional attitude and how it changes depending on situational circumstances. It was also shown that low levels of education increase the risk of discrimination, and can lead to lower levels of health literacy, which spans a number of issues. This was demonstrated through unfamiliarity with entitlements or rights as citizens and unawareness of how the 
health system works and how to navigate it. The consequences of general illiteracy were described as an inability to read health information leaflets, posters or similar materials often displayed in healthcare establishments as well as instructions on prescriptions. Despite a number of the family members being illiterate, they did not identify this barrier, only staff members highlighted it. One family participant did highlight how her lack of education left her unable to dial the emergency services for an ambulance whilst in labor.

"So not knowing how the system works, that would be 1, and then 2, not knowing if they're given a diagnosis, not knowing how to interpret that."

However, some family participants knew their entitlements and how the health system worked. Others were unsure of their entitlements because they had no interest in knowing, not because they were unable to find the information.

“I'm not very sure. I didn't ask, I wasn't curious."

\section{Psychological barriers}

In addition to the three themes (financial, structural and cognitive) described, a further theme of psychological barriers emerged during inductive thematic analysis. Four main subthemes within this included: mistrust, hopelessness, fear and anxiety. These added another dimension of an emotional impact to the community's problems.

Both negative and positive personal experiences shaped the views and mentality community members have of the city and its healthcare services. The attitudes of healthcare staff have led many participants to believe all healthcare practitioners are disrespectful. As such, they are shocked if they are treated otherwise and defensive mechanisms are prominent when interacting with healthcare professionals. This mentality causes mistrust and impairs creating a positive doctor-patient relationship. Mistrust and lack of faith also emerged about the healthcare system, its doctor's and diagnoses. Participants described misdiagnosis, discrepancy between diagnoses depends who they asked and the same diagnosis constantly given for different people.

Furthermore, community members were shown to have a preconceived negative view of healthcare services and staff in this area, discouraging family 
members from accessing care believing only poor care is available. Hopelessness for gaining help was seen as family members are aware, they need help but have no opportunity to, for example, if they are uninsured. This hopelessness extended to the healthcare system; people hope for a change in the system, but are not optimistic about any reform.

"Yes, I would like to, but I have no chance. (...) I don't feel okay, I don't feel good. I don't think I have a chance."

Added to this, some family members are frightened or anxious about seeking a diagnosis because they fear, they have no chance of gaining access to a doctor or subsequent treatment needed. Fear of being diagnosed with a life changing illness also leads to a failure to seek healthcare, because what follows the diagnoses is deemed to be out with their control.

"I am afraid they will discover some other secondary issues ... I don't want to find out. I would rather like die suddenly and not know."

Finally, complexity in the operation of, and corruption within, the healthcare system created psychological barriers of stress and anxiety. Bribery was an issue for the participants and so is a barrier that disregards vulnerability. Anxiety was also induced due to the current staffing shortages and the impact that had on available specialists and care.

"I don't know what to do exactly, I don't know how to, just because I don't want to be treated badly, I want to bribe them, bribe the doctor, but I don't know exactly what to do, and that's my dilemma and my anxiety." 


\section{CONCLUSION AND RECOMMENDATION}

\section{CONCLUSION}

After the researchers qualifying the data analysis, they came up with a vital result that is intimate on improving the perception of the respondents and how to aid the situation. The conclusion was a primary response for an effective communication. The following themes presented were Financial Barriers, Structural Barriers, Cognitive Barriers, and Psychological Barriers. The mentioned barriers followed by a sub-theme that specifically introduced factors on each theme.

The researchers arrived at a conclusion that the government needs to improve the quality of health services for those respondents who are financially challenged. Also, the respondents need to provide equal attention to both priorities such as hospital bills and other home necessities. Then, to those respondents who do not have respective jobs should manifest their concerns to the government; otherwise, the government will not be able to comprehend their needs. Respondents who are financially challenged are also in demand of stabilizing the availability of services in terms of poor social classes. Furthermore, the government must take their responsibilities seriously to avoid damaging their reputation and disobeying their political oath.

The government must widen their accessibility of health services such that a service must cater all medical conditions whether the patient is in critical condition or light condition. The public hospitals, which are under the government, should be informed that it needs to produce an adequate manpower in order to have an organized and immediate service. In order for the hospital to serve the patients regularly, they should have systematic process that would obtain the patients' ideal service they need. Thus, they need to have equal distribution of service to all ages (from a child to an adult).

The authorized personnel on different hospitals should imply the basic manners and humane characteristics in engaging with the patients. Thus, 
personnel should settle an understanding environment for the patients. By means of communicating with the patients, the personnel should set aside their biases and prejudices in accommodating them. Also, in providing a sense of learning, the personnel should simplify their explanation for the patients to be literate enough.

The respondents should avoid a kind of reasoning that inhibits them to think that medical experts are incapable of doing their work efficiently. They must try to alleviate the negative thoughts and senses about the medical society to avoid future complications. In this way, the two can have healthy communication in terms of health services. The application of having a healthy communication could change the perception of respondents towards the medical experts since they starting to trust them, and also for the medical experts, they can exchange reaction to the respondents and will be able to profess a comforting environment.

\section{RECOMMENDATION}

The researchers would like to implicate further improvement for the future researchers in this study. This is to create more impact for the people, and for the government, which is to provide a better accessible health services. To the future researchers, in order for them to be more successful in conducting this research, they need to keep guided in these following recommendations:

- To acquire more valid information, they need to procure five or more participants.

- To secure the availability of each participant, they should plan ahead of time to circumvent future delay of schedule on an interview.

- To use more precise and credible source that would support the study.

- To find additional previous studies and crucial theories that would support the study. 


\section{REFERENCES}

Aftab, W. (2018). Exploring health care seeking knowledge, perceptions and practices for childhood diarrhea and pneumonia and their context in a rural Pakistani community. Retrieved from https://ecommons.aku.edu/pakistan_fhs_mc_chs_chs/343/

Alipio, M. (2020). Predicting Academic Performance of College Freshmen in the Philippines using Psychological Variables and Expectancy-Value Beliefs to Outcomes-Based Education: A Path Analysis. https://doi.org/10.35542/osf.io/pra6z

Alipio, M. (2020). Academic Adjustment and Performance among Filipino Freshmen College Students in the Health Sciences: Does Senior High School Strand Matter?. https://doi.org/10.35542/osf.io/xq4pk

Bogdan, R. and Taylor S. (2019) Introduction to qualitative research methods : a phenomenological approach to the social sciences. Retrieved from https://www.researchgate.net/publication/44435469_Introduction_to_qualit tive_research_methods_a_phenomenological_approach_to_the_social_sa ciences_Robert_Bogdan_and_Steven_J_Taylor/amp

Crosta, P. ( 2017, November 27). What you should know about pneumonia. Retrieved from https://www.medicalnewstoday.com/articles/151632.php

Deborah S.K, T. (2017). Disease mapping for informing targeted health interventions: childhood pneumonia in Bohol, Philippines. Retrieved from onlinelibrary. wiley.com > tmi. $12561>$ pdf

De Vos, J. (1998). Wiredspace.wits.ac.za. Retrieved from http://wiredspace.wits.ac.za/bitstream/handle/10539/1489/03Chapter3.pdf? sequence $=6$

Ford, J., Jones, A. and Steel, N. (2015, September 16). Improving access to high-quality primary care for socioeconomically disadvantaged older people in rural areas: a mixed method study protocol. Retrieved from https://www.ncbi.nlm.nih.gov/pmc/articles/PMC4577946/\#!po=73.8095

Glantz et al. (2002). Interpersonal Communication and Relations | Social Cognitive Theory. Retrieved from https://www.utwente.nl/en/bms/communication-theories/sorted-by- 
cluster/Interpersonal-Communication-and-Relations/social-cognitivetheory/

Johnson, M. and Silva, M. E (2009). The Davao City Health System: An Approach to Optimally Locating Community Health Facilities. Retrieved from http://citeseerx.ist.psu.edu/viewdoc/summary?doi=10.1.1.541.3277

Kathiri, N. (2010). ResearchGate | Share and discover research. Retrieved from https://www.researchgate.net/

Kosai, H., Oshitani, H. and Tamaki, R. (2015). Incidence and Risk Factors of Childhood Pneumonia-Like Episodes in Biliran Island, Philippines-A Community-Based Study. Retrieved from https://www.ncbi.nlm.nih.gov/pmc/articles/PMC4418693/

LaMorte, W. (2018). The Health Belief Model. Retrieved from http://sphweb.bumc.bu.edu/ottt/MPH-

Modules/SB/BehavioralChangeTheories/BehavioralChangeTheories2.html

Langkos, S. (2019) Chapter 3 - Research Methodology: Data collection method and Research tools. Retrieved from https://www.academia.edu/10092020/CHAPTER_3__RESEARCH_METHODOLOGY_Data_collection_method_and_ tools

Lincoln, YS. \& Guba, EG. (1985). Techniques for establishing credibility. Retrieved from http://www.qualres.org/HomeLinc-3684.html

Massachusetts General Hospital. (2014, February 20). Rural primary care physicians are committed to professionalism, quality improvement. ScienceDaily. Retrieved March 14, 2019 from www.sciencedaily.com/releases/2014/02/140220112509.htm

Matovu, F., Nanyiti, A. and Rutebemberwa, E. (2014). Household health careseeking costs: experiences from a randomized, controlled trial of community-based malaria and pneumonia treatment among under-fives in eastern Uganda. Retrieved from https://www.ncbi.nlm.nih.gov/pmc/articles/PMC4059171

Rashid, S., Hadi, A., Afsana, K., and Begum, S. ( 2001, December 21). Acute respiratory infections in rural Bangladesh: cultural understandings, practices and the role of mothers and community health volunteers. Retrieved from https://onlinelibrary.wiley.com/doi/full/10.1046/j.1365-3156.2001.00702.x 
